\section{LOW-TEMPERATURE PHYSICS IN NORTH AMERICA}

\author{
By Prof. J. F. ALLEN \\ University of St. Andrews
}

$\mathrm{D}^{\mathrm{n}}$ URING the past summer, an opportunity was taken to visit the principal laboratories in the United States and Canada which are engaged in lowtemperature research, with the exception of the Naval Research Laboratory in Washington, the laboratory directed by Giauque at Berkeley and that planned by Long at Chicago.

Prior to the War, comparatively little interest was shown in America in cryogenic research, with the notable exceptions of the Chemical Laboratory at Berkeley and the MeLennan Laboratory at Toronto, and on the whole what interest there was lay in the field of chemical thermodynamics. To-day, although the position is changing rapidly, and again with the possible exception of Berkeley, there are still no eryogenic laboratories in America such as we know them on the eastern side of the Atlantic, equipped to carry out physical research in all the principal lowtemperature fields, of which the three most important are superconductivity, paramagneties and the properties of liquid helium. But the position is changing very rapidly indeed. There is in America a growing realization of the importance of low-temperature physics, and a very rapid growth in the number of laboratories using liquid helium. There are roughly a dozen such at the present time, and another dozen will probably be added within the next year.

This extremely rapid growth has been made possible largely by the new liquefier designed by Collins of the Massachusetts Institute of Technology. This liquefier can be considered as deriving from the one designed by Kapitza, but it is carried a stage further in that all the cooling is obtained by the principle of external work, and no other refrigerant, such as liquid air or hydrogen, is required. There are in the Collins machine two expansion cylinders and pistons, one operating at roughly the temperature of liquid air and the other at the temperature of liquid hydrogen. There is essentially only one heat exchanger, which is tapped off at two points to feed the cylinders, and at the bottom end is the JouleThomson valve where liquefaction is performed. The whole apparatus is extremely simple and elegant. It has been designed for something like mass production and can now be purchased, more or less 'over the counter', from the Arthur D. Little Corporation of Cambridge, Mass., for 18,000 dollars (August 1947 price). For this, it was learned, one obtains the complete plant, including gas holder, garage-type compressor, helium purifier and auxiliary vacuum pump, installed and ready to run. The whole assembly, less the gas holder and compressor, is fitted into a rectangular box about $5 \mathrm{ft} .6$ in. in height, $3 \mathrm{ft} .6$ in. wide and $3 \mathrm{ft}$. deep. The machine takes about four hours to cool to helium temperature and will then produce somewhat less than two litres of liquid helium an hour. It is not limited to helium production, but by its nature it will liquefy any gas. If, however, other liquid gases are wanted, it is possible to use helium as the working substance and liquefy other gases by contact, and it can thus provide a particularly useful and safe way of making liquid hydrogen.

The Collins liquefier, being compact, self-contained and simple to operate, is an admirable liquefier for the laboratory in which it is desired simply to extend existing researches or measurements to the helium range of temperatures. Also, it is designed expressly for the purpose of having experimental apparatus installed directly in the liquefier, since there is an opening in the top some 5 in. in diameter and a clear space beneath at least as large leading directly to the liquid helium bath. These reasons, together with the fact that the U.S. Navy makes extremely generous provisions for physical research in America, produce a tendency in the more ambitious laboratories to have a separate Collins machine for each experiment. Both the Naval Research Laboratory in Washington and the Massachusetts Institute of Technology have two liquefiers, and it was understood that both laboratories were planning to acquire additional ones.

It is, of course, easily possible to decant the liquid helium from the Collins machine; but that requires additional equipment such as Dewar vessels and liquid air for the thermal shielding, which are not always available. Hence the liquefier tends to be used for experiments which do not require either visual observation through the apparatus or the presence of magnetic fields. Furthermore, due to the comparatively poor shielding of the liquid in the liquefier from room-temperature radiation, it is not easy to reach a very low temperature by reducing the vapour pressure of the liquid.

Two other helium liquefiers which are being constructed are of interest. In Toronto an attempt is being made to use a turbine as a means of extracting work, and in the Ohio State University at Columbus a very large output of liquid helium is expected from a Joulo-Thomson liquefier using only liquid hydrogen, of which there is a plentiful supply, as a refrigerant. A feature of the laboratory at Columbus was the immense size of the cryostats. The glass Dewar flasks, made on the premises, were four to five feet in height and six to eight inches in diameter.

Several other laboratories have small Linde or Simon single-expansion liquefiers, and at Yale there is a more or less exact replica of the Kapitza liquefier in the Royal Society Mond Laboratory at Cambridge.

of the low-temperature research which is going on there is, of course, a considerable amount of accurate calorimetry devoted to chemical thermodynamics. On the physical side, the most important subjects at the moment are the separation and properties of the isotope helium 3- and the behaviour of superconductors under the action of very highfrequency currents, using radar techniques.

The phenomenon of the disturbance of superconductivity by high-frequency fields was first observed by H. London in Bristol before the War, and the effect of using $25-\mathrm{cm}$. waves in superconductors and in normal metals has been examined in detail by Pippard in Cambridge sinee the War. At the Massachusetts Institute of Technology, under Slater, and at the Naval Research Laboratory in Washington, the problem is under investigation using $1 \cdot 25-\mathrm{cm}$. waves. The supereonductors which have been investigated so far are tin and lead, and the methods which are employed are slightly different in the two laboratories. At the Massachusetts Institute of Technology, the waves, conducted in by wave-guide, are reflected at the end of the guide by a superconducting element which is in the form of a cavity of the dimensions of the guide, across which a thin perforated superconducting membrane is stretched. At the Naval Research Laboratory the 
waves are conducted down to a superconducting drum-shaped cavity and the energy absorption is measured by the determination of the ringing time of the cavity.

Slater has obtained results similar to those of Pippard, but the destruction of superconductivity was more complete, as was to be expected from the higher frequency which has been used. He is also studying the so-called anomalous skin resistance of normal metals at low temperatures.

The work on the properties of the rare isotope helium 3, which has a normal concentration of about one part in $10^{6}$ of helium 4, has been done by Daunt at Columbus and Lane at Yale with the massspectrographic collaboration of Nier at Minnesota. Lane has found that the vapour above the surface of liquid helium has a higher concentration of helium 3 than has the liquid, which indicates that if pure helium 3 can exist as a liquid, then its normal boiling point will be lower than that of helium 4. Daunt has further shown that helium 3 does not participate in the surface transfer of liquid helium II, which means that helium 3 is not in the superfluid state, at least at temperatures above $1 \cdot 5^{\circ} \mathrm{K}$., whereas helium II loses its superfluidity at $2 \cdot 19^{\circ} \mathrm{K}$. The fact that helium 3 is not superfluid offers the possibility of considerable enrichment of the helium 3 content by 'straining' helium II through a porous plug-called by the Americans a 'superleak'. It was rumoured in America that there exists a small quantity, of the order of 1 c.c., of pure helium 3, but not produced by the above means.

Other research on helium II at Yale consisted in investigations on second sound, in which, among other things, an attempt was being made to convert ordinary sound into second sound by passage of the waves through a porous membrane. At the Massachusetts Institute of Technology, Squire has made an investigation of the attenuation of ordinary sound, of ultra-sonic frequency, in liquid helium. Here the most important feature is the fact that the absorption rises to an indefinitely high maximum at the $\lambda$-point.

Among other researches in progress, Andrews at Johns Hopkins is investigating the phenomenon which he has discovered of the 'rectifying' property of the transition region in superconducting niobium carbide, which occurs in the neighbourhood of $15^{\circ} \mathrm{K}$. One of the odd features of this phenomenon is that at a suitable temperature and with a suitable biasing current through it, the carbide specimen can pick up the local radio station with the aid of an amplifier and loud-speaker.

Boorse, at Columbia, is also planning to investigate niobium carbide and nitride, but from the magnetic point of view. He has a Simon expansion liquefier. Brickwedde, of the U.S. Bureau of Standards, also has a Simon liquefier of about one quartre-litre capacity. Aside from his calorimetric work, he is investigating the so-called 'overshoot' phenomenon in superconductivity which he discovered some years before the War. Some criticism has been expressed of the reality of this phenomenon, which involves very high measuring currents, owing to the possibility of local temperature fluctuations, and he is planning to repeat the investigations on indium, which has a transition temperature in the helium II region, where temperature fluctuations are much more difficult to produce.

None of the cryogenic laboratories visited are at present engaged on magnetic cooling experiments, although one or two of them intend to commence such work within the next year or two.

\section{TOWARDS THE SYNTHESIS OF CHOLESTEROL}

\author{
By DR. J. W. CORNFORTH and SIR ROBERT \\ ROBINSON, F.R.S.
}

National Institute for Medical Research and Dyson Perrins
Laboratory, University of Oxford

TO development of organic chemistry or of bio1 chemistry has unfolded in a more dramatic manner in recent years than that concerned with the nature and functions of the steroids, the bile acids, and the related vitamins and hormones.

The typical compounds, cholesterol and cholic acid, excited the curiosity of Windaus and of Wieland because of their complex and obviously interesting molecular constitution; and the brilliant researches of these pioneers were supplemented by contributions from many other laboratories, especially when discovery of the biological significance of the group came to light. The chemical puzzle was transformed into a problem of vital urgeney for physiology and medicine. The outcome has been that the structures of the chief members of the rapidly expanding family of substances are known in so far as that is possible without the final confirmation of successful synthesis. This latter task has been regarded as one of the most difficult that could be undertaken by the organic chemist, for the simple reason that it is far from sufficient to devise a method of constructing a substance corresponding to the formula as written on paper, difficult though that may be in itself. The molecule of dihydrocholesterol (I) contains nine asymmetric carbon atoms, and hence 512 stereoisomeric forms are possible. There is reason to believe that all these should be capable of existence. Even if the sidechain is not present, and position- 17 carries a carbonyl group, there are still 128 stereoisomeric modifications. It is a triumph of analysis that a combination of chemical and physical methods, culminating in the X-ray crystallographic studies of cholesteryl iodide ${ }^{1}$, has disclosed the stereochemical configuration of the ring-systems (cf. II) in the bestknown steroids.

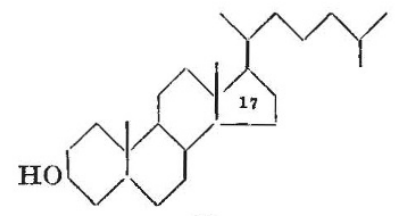

(I)

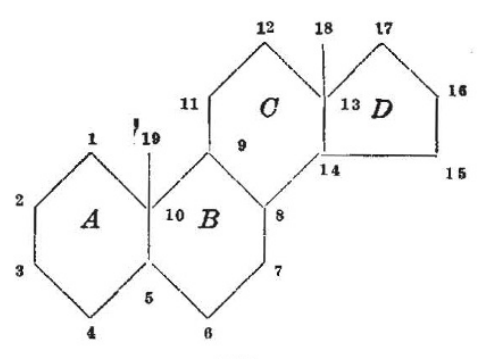

(II)

Rings $A$ and $B$ are fused together in the cis-position (coprosterol, cholic acid) or trans-position (dihydrocholesterol) in various derivatives, whereas rings $B$ and $C$ are fused in the trans-position in all known cases. For any given orientation of rings $A$ and $B$, 